\title{
THE EXTREME-ULTRAVIOLET CONTINUUM OF A STRONG STELLAR FLARE
}

\author{
D. J. Christian, M. Mathioudakis, and D. Jevremović ${ }^{1}$ \\ Department of Pure and Applied Physics, Queens University Belfast, Belfast, BT7 1NN, Northern Ireland; \\ d.christian@qub.ac.uk,m.mathioudakis@qub.ac.uk, d.jevremovic@qub.ac.uk \\ J. DupuIs \\ Johns Hopkins University, Department of Physics and Astronomy \\ AND \\ S. VENNES AND A. KAWKA \\ Department of Mathematics, Australian National University, Canberra, ACT 0200, Australia \\ Received 2003 June 4; accepted 2003 July 7; published 2003 July 18
}

\begin{abstract}
We present the serendipitous detection of an extreme-ultraviolet flare on EUVE J0613-23.9B. The flare showed over a 200-fold increase above the quiescent emission in the DS/Lexan 60-200 $\AA$ wavelength band. Optical spectroscopy revealed that the event was associated with an active dM3.5e star. The EUVE spectra are dominated by emission lines formed at temperatures in excess of $10^{7} \mathrm{~K}$. The observation is unique as we have detected, for the first time, a strong Lyman continuum in the EUVE long-wavelength range (320-650 $\AA$ ). The flare in the continuum $(T \approx 20,000-30,000 \mathrm{~K})$ was extremely short, lasting for less than $500 \mathrm{~s}$, while in the DS $\left(T \approx 10^{7} \mathrm{~K}\right)$ its duration was $\approx 28 \mathrm{ks}$. The total energy of the flare in the DS is $\sim 3 \times 10^{34} \mathrm{ergs}$. We have made a fit to the continuum using semiempirical model atmospheres and derived the time-averaged temperature and density structures.
\end{abstract}

Subject headings: stars: flare — stars: late-type — ultraviolet: stars

\section{INTRODUCTION}

The extensive surveys of the X-ray sky over the past 30 years revealed that in almost one-third of the sources discovered the emission is of coronal origin (Schmitt 1997). This fraction is considerably higher in the extreme ultraviolet, where $50 \%$ of the sources are late-type stars. EUVE observations allowed detailed spectroscopic studies of flares on several cool stars including AU Mic, AD Leo (Cully et al. 1994, 1997), and EQ Peg (Monsignori-Fossi et al. 1995). Despite the extensive wavelength coverage of EUVE (70-760 $\AA$ ), the flares have been detected primarily in the 70-300 $\AA$ range. These flares are best pronounced in the coronal lines of highly ionized iron (Fe XXI-Fe XXIV) and in the short-wavelength continuum

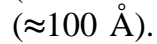

The extreme-ultraviolet (EUV) continuum (300-912 A) in late-type stars is generated in the chromosphere and transition region. Semiempirical models of active stellar atmospheres show that the radiated energy is a strong function of the transition region pressure and can provide information of the plasma properties in this region. The importance of the EUV Lyman continuum of late-type stars in the atomic equilibrium (especially during stellar flares) has been emphasized; however, no observations of stellar flares for EUV wavelengths longer than $\approx 300 \AA$ have been reported in the literature (Houdebine et al. 1996). This is primarily due to the low sensitivity of the instruments, weak fluxes, and strong interstellar medium absorption in wavelengths below the Lyman limit.

In this Letter, we present the serendipitous detection of a strong flare on an active $\mathrm{M}$ star during an EUVE observation of the G star HD 43162. Our observations show the detection of continuum emission extended to wavelengths as long as $650 \AA$. In $\S 2$ we present the EUVE observations and followup optical spectroscopy. In $\S 3$ we examine the temporal be-

\footnotetext{
${ }^{1}$ Astronomical Observatory, Volgina 7, 11160 Belgrade, Serbia and Montenegro.
}

havior of the flare, derive the flare energy, and use the continuum to derive the time-averaged temperature and density structure of the atmosphere. Concluding remarks are given in $\S 4$.

\section{EUVE AND OPTICAL OBSERVATIONS}

The Extreme Ultraviolet Explorer (EUVE) obtained 14 observations of HD 43162 between 2000 October 20 and December 8 as part of a Guest Observer program (PI: E. Gaidos). The observations were carried out with the Deep Survey/Spectrometer telescope (DS/S). Three of the telescope segments provided light for the main imager with a central Lexan/B filter (60-200 $\AA$ ), while the remaining three provided light for the short-wavelength (SW, 70-190 A), medium-wavelength (MW, 140-380 A), and long-wavelength (LW, 280-760 A) spectrometers (Bowyer \& Malina 1991). Following aspect verification and exposure time corrections, a total of $180 \mathrm{ks}$ of data were accumulated. We obtained the EUVE data files of the HD 43162 field from the Multimission Archive (MAST). An inspection of the DS images revealed a second source (R.A. = $06^{\mathrm{h}} 13^{\mathrm{m}} 47^{\mathrm{s}} .2$, decl. $=-23^{\circ} 54^{\prime} 26^{\prime \prime}$; EUVE J0613-23.9B) located 2.5 southeast of the Guest Observer target. At this position off-axis, the point-spread function of the DS is still optimal, and there is no source confusion for EUVE J0613-23.9B and HD 43162. The source is not close to the edge of the detector and remains unaffected by vignetting (see Figs. 3, 6, and 7 of Sirk et al. 1997). We converted these data products into QPOE (quick position oriented event) files for the Deep Survey and spectrometer data. DS QPOE files were used to derive light curves for both sources (as described in Christian 2002 and references therein). Spectrometer QPOE files were used to perform spectral extractions as a function of time.

A search of SIMBAD revealed no cataloged sources within $60^{\prime \prime}$ of EUVE J0613-23.9B. We have obtained optical spectra for all the likely candidates located within the DS positional uncertainty circle. The optical observations were conducted on 


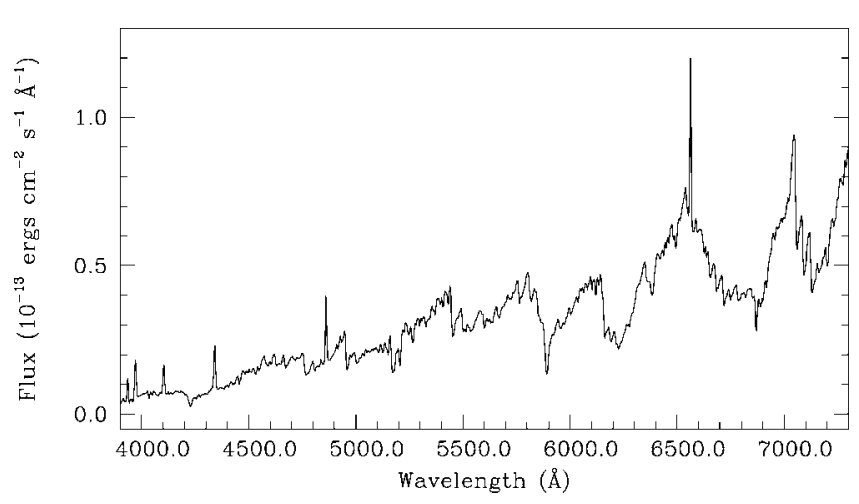

FIg. 1.-Optical spectrum of EUVE J0613-23.9B

2002 September 14 using the Cassegrain spectrograph and the 74 inch telescope at Mount Stromlo Observatory. We used the 300 line $\mathrm{mm}^{-1}$ grating blazed at $5000 \AA$ and the $2 \mathrm{k} \times 4 \mathrm{k} \mathrm{CCD}$ camera binned $2 \times 2$. The spectra cover the wavelength range $3900-7500 \AA$ with a spectral resolution of $\approx 8 \AA$. The spectra were analyzed using standard routines within IRAF.

Absolute flux calibration was established with multiple spectra of the white dwarf star Feige 110 observed with the same instrumental setup and under similar observing conditions. The most likely optical counterpart of EUVE J0613-23.9B is an active $M$ dwarf with the Balmer series in emission and an $\mathrm{H} \alpha$ equivalent width of $6.0 \AA$. Its optical spectrum is shown in Figure 1.

We have used the strength of the $\mathrm{TiO}$ bands to derive a spectral type of $\mathrm{dM} 3.5 \mathrm{e}$ and absolute magnitude of $M_{V}=$ 11.9 for this source (Reid, Hawley, \& Gizis 1995). An apparent magnitude of $m_{v}=12.7$ was calculated from the spectra using the SBANDS routine within IRAF. These parameters give a distance of $15_{-4}^{+5} \mathrm{pc}$.

The 0.5 mag uncertainty in the absolute magnitude derived from the TiO band (Reid et al. 1995) dominates the uncertainty in $m_{v}$, which we conservatively estimate at $0.2 \mathrm{mag}$.

\section{RESULTS AND DISCUSSION}

\subsection{Flare Spectra and Light Curves}

In Figure 2 we present the DS and spectrometer light curves of EUVE J0613-23.9B from the 2000 October 22 observation. A very strong flare with a peak count rate of $3.4 \pm 0.2$ counts $\mathrm{s}^{-1}$, over a factor of 200 above the preflare quiescent emission of $0.013 \pm 0.002$ counts $\mathrm{s}^{-1}$, is detected. The flare rise time is less than $1 \mathrm{ks}$, and the decay time is $\sim 28 \mathrm{ks}$. The decay $e$ folding timescale is short, at $\approx 3 \mathrm{ks}$.

We note, for comparison, that the EUV flares on AU Mic and EUVE J1438-43.2 showed an increase by a factor of $\approx 15$ above the quiescent emission (Cully et al. 1993; Christian \& Vennes 1999).

Using the DS light curve as a guide, we extracted a spectrum from the SW and MW spectrometers for a $3 \mathrm{ks}$ time bin that included the orbits of the flare peak and decay phase (first two orbits in Fig. 2a). These spectra are shown in Figure 3. Emission lines of the highest ionization stages of iron (Fe XXII-XXIV) are detected in the spectra. These lines are typical of active stellar coronae.

Fe XXI lines in the EUV provide excellent density diagnostics for hot coronal plasmas (Keenan et al. 2001). Although the Fe XXI $128.73 \AA$ line is one of the strongest in the spectra of hot coronal sources, it has not been detected in this case. Its

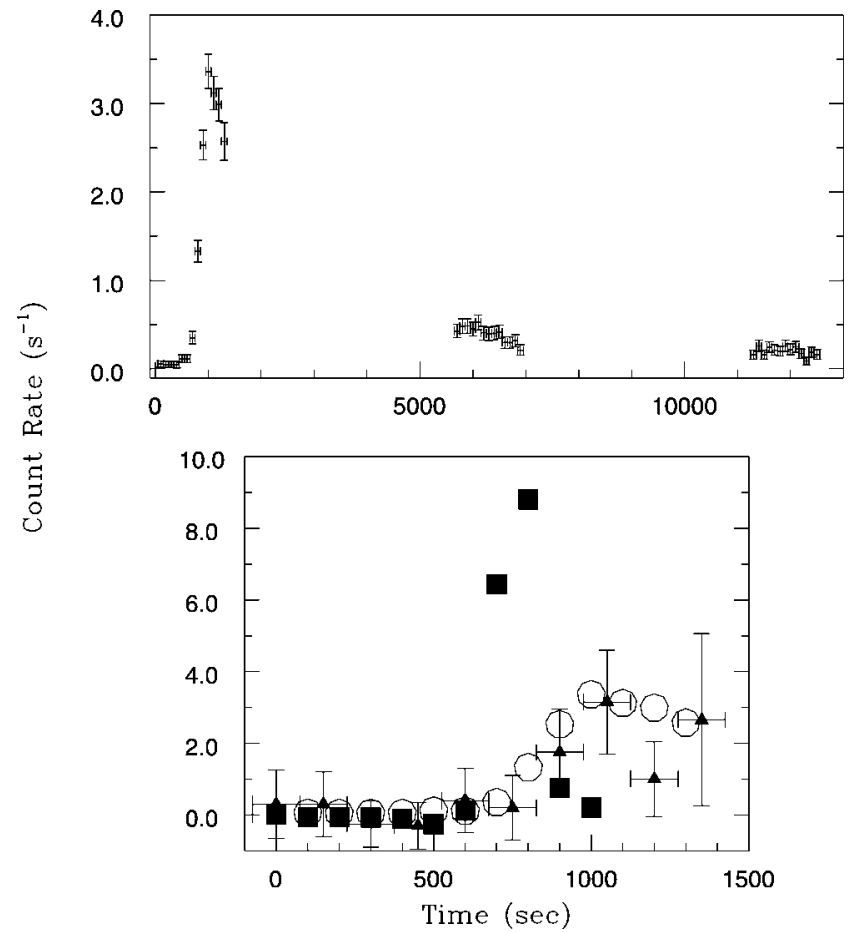

FIG. 2.-EUVE light curves for the flare on EUVE J0613-23.9B. Time zero for both panels corresponds to MJD 51,839.89115. (a) The EUVE DS Lexan/B light curve. The preflare quiescent emission was observed for 13 EUVE orbits and had an average count rate of 0.013 counts s ${ }^{-1}$. (b) Expanded DS and spectrometer light curves. The DS light curve is plotted as open circles, the LW spectrum is plotted as filled squares, while the Fe XXIV line is overplotted as filled triangles with error bars. The error bars for the DS and LW spectra are similar to the symbol size. Bin sizes are $100 \mathrm{~s}$ for DS and LW, and $150 \mathrm{~s}$ for Fe XXIV. The Fe XXIV count rate has been multiplied by a factor of 50 for scaling.

emissivity is decreasing, by as much as a factor of 3 , with increasing electron density (Brickhouse, Raymond, \& Smith 1995). The Fe XxII $135.78 \AA$ line with a lower emissivity is detected in the spectrum. Since Fe XXI and Fe XXII are formed at approximately the same temperature $\left(10^{7.1} \mathrm{~K}\right)$, we conclude that the nondetection of Fe XXI is due to an increased electron density $\left(n_{e}>10^{12} \mathrm{~cm}^{-3}\right)$. A similar approach to the estimate of electron density using Fe XXI has been adopted by Griffiths \& Jordan (1998).

One of the most conspicuous features in the spectra is the

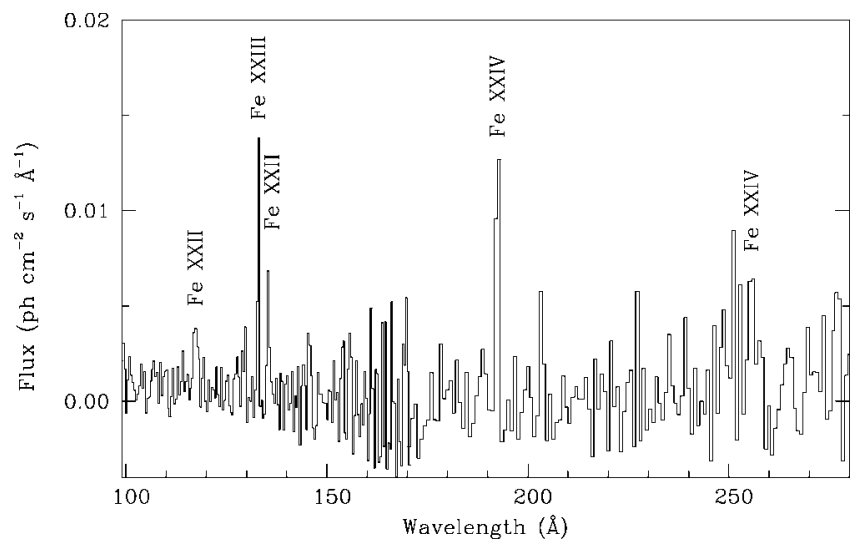

FIG. 3.-Short- and medium-wavelength spectra of the flare on EUVE J0613-23.9B. The spectra were extracted from the first two orbits shown in Fig. $2 a$. The most prominent emission lines are identified. 
TABLE 1

Line and CONTINUUm FluXes for Flare

\begin{tabular}{|c|c|c|}
\hline ID & $\lambda_{\mathrm{lab}}^{\mathrm{a}}$ & Flux $_{\text {cor }}{ }^{b}$ \\
\hline$\ldots \ldots \ldots \ldots \ldots$ & 108.370 & 0.002 \\
\hline Fe xxII $\ldots \ldots \ldots \ldots \ldots \ldots$ & 117.167 & 0.007 \\
\hline Fe xxiII $\ldots \ldots \ldots \ldots \ldots$ & 132.831 & 0.013 \\
\hline Fe XXII $\ldots \ldots \ldots \ldots \ldots \ldots$ & 135.774 & 0.008 \\
\hline Fe XXIV $\ldots$ & 192.042 & 0.025 \\
\hline Fe XXIV & 255.102 & 0.008 \\
\hline Continuum $\ldots \ldots \ldots \ldots \ldots$ & $(320-650 \AA)$ & 28.2 \\
\hline
\end{tabular}

${ }^{\text {a }}$ Laboratory wavelength.

${ }^{\mathrm{b}}$ Corrected (intrinsic) flux in units of $10^{-10}$ ergs $\mathrm{cm}^{-2} \mathrm{~s}^{-1}$ for a $N_{\mathrm{H}}$ of $1.0 \times 10^{18} \mathrm{~cm}^{-2}$.

strong EUV continuum seen longward of $320 \AA$. We will return to the continuum later. Integrated line fluxes for the strongest lines and the continuum are given in Table 1.

We have not detected any significant signal in the quiescent spectra of EUVE J0613-23.9B.

We constructed light curves from specific spectral regions and the strongest emission lines. Although the sensitivity of the DS/Lexan is a factor of 10 higher than the SW spectrometer, they cover the same spectral range and their light curves are identical. Light curves of individual lines in the SW and MW spectrometers (e.g., Fe XXIII, Fe XXIV) have approximately the same shape as the DS. This is expected as they sample approximately the same temperature $\left(\approx 2 \times 10^{7} \mathrm{~K}\right)$. In Figure $2 b$ we show the flare light curves for the DS/Lexan, LW continuum (320-650 $\AA$ ) and the Fe XxIV (192 $\AA$ ) line. The flare in the LW spectrometer is extremely short with a rise to peak in $\approx 100 \mathrm{~s}$ and a decay of $\approx 200 \mathrm{~s}$. This compares with a rise time of $\approx 1 \mathrm{ks}$ and a decay in excess of $\approx 28 \mathrm{ks}$ for the DS.

\subsection{Flare Energy}

Fluxes from the count rates have been determined using the Monsignori-Fossi \& Landini (1994) line emissivities for a coronal temperature of $10^{7} \mathrm{~K}$. An interstellar hydrogen column density of $10^{18} \mathrm{~cm}^{-2}$, implied by the distance to EUVE J0613-23.9B and measurements of the local ISM distribution, has been adopted. ${ }^{2}$

We determined observed fluxes for the Lexan emission of $1.7 \times 10^{-13} \mathrm{ergs} \mathrm{cm}^{-2} \mathrm{~s}^{-1}$ in quiescence and $4.1 \times 10^{-11} \mathrm{ergs}$ $\mathrm{cm}^{-2} \mathrm{~s}^{-1}$ for the flare peak. Using the distance derived above of $15 \mathrm{pc}$ gives a luminosity for the quiescent Lexan EUV flux of $4.6 \times 10^{27} \mathrm{ergs} \mathrm{s}^{-1}$. We calculated a peak flare luminosity in the Lexan band of $1.1 \times 10^{30} \mathrm{ergs} \mathrm{s}^{-1}$ and estimate the total flare energy in the Lexan band (60-200 $\AA$ ) to be of order $3 \times 10^{34}$ ergs. This energy is integrated over the duration of the entire event $(28 \mathrm{ks})$. The flare energetics are similar to the large EUV flare detected from AU Mic in 1992 (Cully et al. 1993 ) and $\approx 50 \%$ higher than other EUV-detected flares from late-type stars.

\subsection{The EUV Continuum}

Theoretical modeling of solar/stellar quiescent and flare atmospheres has revealed that the EUV continuum can provide important constraints on the upper chromosphere and transition region (Vernazza, Avrett, \& Loeser 1981; Ding \& Schleicher 1997; Abbett \& Hawley 1999). Despite over 200 spectroscopic observations of cool stars over the 8 years of the EUVE mission, there are no reports of continuum detection longward of

${ }^{2}$ See http://archive.stsci.edu/euve/ism/ismform.html.

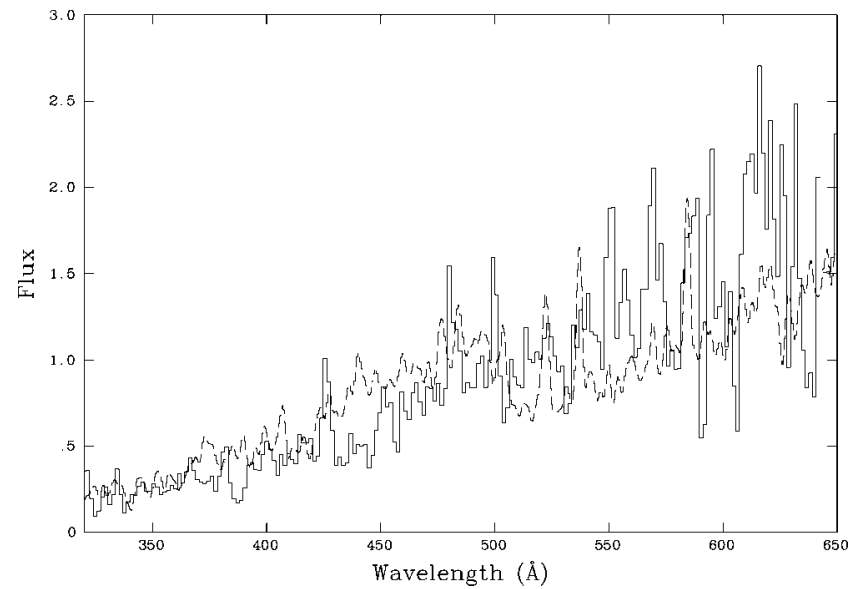

FIG. 4.-LW continuum observed during the flare on EUVE J0613-23.9B (solid line). The spectrum has been extracted with a $400 \mathrm{~s}$ time bin. A model spectrum derived using the temperature structure shown in Fig. 5 is superposed (dashed line).

$300 \AA$. Even in the solar case observations of the EUV continuum are scarce (Dupree et al. 1973). The continuum detected here is very strong, with a luminosity of $8 \times 10^{31} \mathrm{ergs} \mathrm{s}^{-1}$ integrated over the 320-650 А wavelength range.

The continuum was studied further by calculating semiempirical models of stellar atmospheres using the general stellar atmosphere code PHOENIX (Hauschildt et al. 1999), adopted for use with chromospheric models (Doyle et al. 2001; Mihajlov et al. 2003). PHOENIX solves simultaneously the equations of hydrostatic equilibrium, multilevel, and multispecies statistical equilibrium and NLTE radiative transfer in spherical geometry. We have built a grid of chromospheric models with an effective temperature of $3300 \mathrm{~K}$ and a gravity of $\log g=$ 5. The procedure for building the models is similar to the "schematic" one in Doyle et al. (2001) and Jevremović, Doyle, \& Short (2000). We used a range of column masses at the temperature minimum $\left(\log m_{0}=-1\right.$ to 0$)$ and the bottom of the transition region (TR) $\left(\log m_{\mathrm{tr}}=-3\right.$ to -0.7$)$. Considering the higher gravity and lower effective temperature of our models, they are in broad agreement with the results of Hawley \& Fischer (1994) and Abbett \& Hawley (1999), which derive similar temperature structures with flare heating mechanisms that include accelerated electrons and soft X-ray irradiation. In all NLTE calculations, we have used a model atom for $\mathrm{H}$ i that includes the lowest 30 levels plus continuum, while for He I we have considered all levels with $n \leq 4$ (19 levels) plus continuum. The collisional data for hydrogen are those from Vriens \& Smeets (1980), and for helium from Berrington \& Kingston (1987) and Lanzafame et al. (1993). The temperature at the bottom of the TR was allowed to vary from 8000 to $20,000 \mathrm{~K}$ as was the temperature rise in the TR $(d \log T / d \log m$ ranged from -8 to -4 ).

The model parameters that produce the best fit to the continuum are $\log m_{0}=0, \log m_{\mathrm{tr}}=-1.7, T_{\mathrm{tr}}=18,000 \mathrm{~K}$, and $d \log T / d \log m=-5$. The model spectrum was then adjusted to the resolution of the LW spectrometer, and Poisson noise was added to it (Fig. 4). The electron temperature and density structures derived are shown in Figure 5. The electron density that we determine for the middle chromosphere is relatively high at around $2 \times 10^{15} \mathrm{~cm}^{-3}$. Very high chromospheric electron densities, in the range of $10^{14}-10^{15} \mathrm{~cm}^{-3}$, have been encountered in stellar flares before (Worden et al. 1984; Jevremović et al. 1998). 


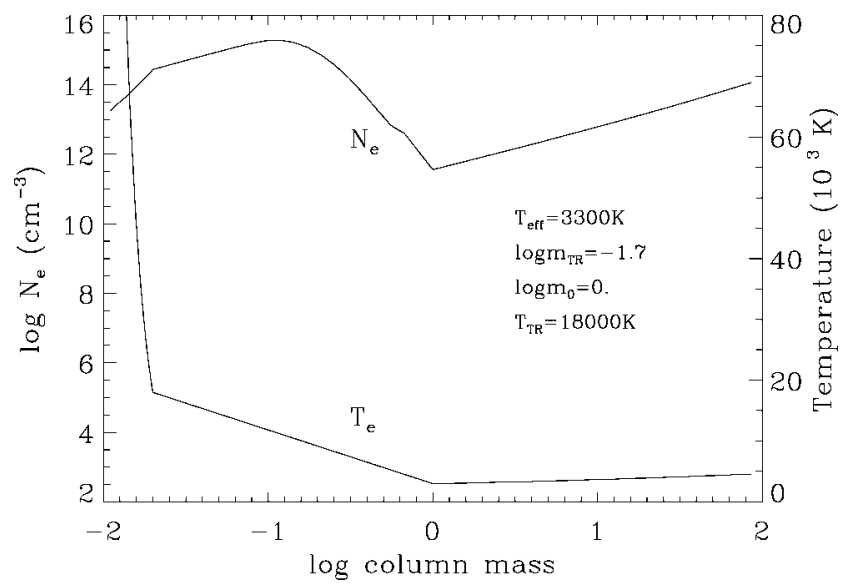

FIG. 5.-Average temperature and density structure of the flare on EUVE J0613-23.9B.

The ratio of the continuum fluxes between the blue and red sides of the Balmer jump, as determined from our model spectrum, is less than $5 \%$. This very small difference is consistent with the nondetection of the Balmer jump in most stellar flares (see Jevremović et al. 1998; García-Alvarez et al. 2002). The continuum at the Lyman jump shows an increase of a factor of $\approx 10$, but as there are no flare observations at this wavelength this prediction cannot be tested. The spectral distribution of the continuum radiation peaks at $2400 \mathrm{~A}$. This compares with a spectral distribution that peaks at $3500 \AA$ for the 1985 flare on AD Leo (Hawley \& Pettersen 1991).

\section{CONCLUSIONS}

The short-wavelength continuum $(\lambda \sim 100 \AA)$ is a common feature in the EUVE spectra of active stars and can provide temperature information in the corona $\left(T>10^{6.5} \mathrm{~K}\right)$. In this Letter we have presented the first detection of the Lyman continuum in a late-type star for wavelengths longer than $300 \AA$. The continuum was detected during a stellar flare on EUVE J0613-23.9B. The observations combined with semiempirical models of stellar atmospheres allowed us to derive the temperature and density structure in the upper chromosphere and transition region. The strong recombination continuum that we see in the EUV is consistent with the high value estimated for the chromospheric electron density. Although some of the parameters derived may be considered as high, our findings are in general agreement with earlier observations of stellar flares. Note that with a 200 -fold increase over the quiescent state, this is one of the most extreme events ever observed. We emphasize that as the flare is a highly dynamic event, the atmospheric structure will change dramatically as a function of time. Given the limited time resolution of $\approx 400 \mathrm{~s}$, the derived structure is representative of the time-average flare event.

This research was partially supported by NASA Astrophysics Data Program grant S-54536-G and the UK Particle Physics and Astronomy Research Council (PPARC). We thank an anonymous referee for suggested improvements to the manuscript.

\section{REFERENCES}

Abbett, W. P., \& Hawley, S. L. 1999, ApJ, 521, 906

Berrington, K. A., \& Kingston, A. E. 1987, J. Phys. B, 20, 6631

Bowyer, S., \& Malina, R. F. 1991, in The EUVE Mission in Extreme Ultraviolet Astronomy, ed. R. F. Malina \& S. Bowyer (Oxford: Pergamon), 391

Brickhouse, N. S., Raymond, J. C., \& Smith, B. W. 1995, ApJS, 97, 551

Christian, D. J. 2002, AJ, 124, 3478

Christian, D. J., \& Vennes, S. 1999, AJ, 117, 1852

Cully, S. L., Fisher, G. H., Abbott, M. J., \& Siegmund, O. H. W. 1994, ApJ, 435, 449

Cully, S. L., Fisher, G. H., Hawley, S. L., \& Simon, T. 1997, ApJ, 491, 910

Cully, S. L., Seigmund, O. H., Vedder, P., \& Vallerga, J. V. 1993, ApJ, 414, L49

Ding, M. D., \& Schleicher, H. 1997, A\&A, 322, 674

Doyle, J. G., Jevremović, D., Short, C. I., Hauschildt, P. H., Livingston, W., \& Vince, I. 2001, A\&A, 369, L13

Dupree, A. K., et al. 1973, ApJ, 182, 321

García-Alvarez, D., Jevremović, D., Doyle, J. G., \& Butler, C. J. 2002, A\&A, 383,548

Griffiths, N. W., \& Jordan, C. 1998, ApJ, 497, 883

Hauschildt, P. H., et al. 1999, ApJ, 525, 871

Hawley, S. L., \& Fischer, G. H. 1994, ApJ, 426, 387

Hawley, S. L., \& Pettersen, B. R. 1991, ApJ, 378, 725
Houdebine, E. R., Mathioudakis, M., Doyle, J. G., \& Foing, B. H. 1996, A\&A, 305, 209

Jevremović, D., Butler, C. J., Drake, S. A., O’Donoghue, D., \& van Wyk, F. 1998, A\&A, 338, 1057

Jevremović, D., Doyle, J. G., \& Short, C. I. 2000, A\&A, 358, 575

Keenan, F. P., Aggarwal, K. M., Williams, D. R., Mathioudakis, M., \& Phillips, K. J. H. 2001, MNRAS, 326, 138

Lanzafame, A. C., Tully, J. A., Berrington, K. A., Dufton, P. L., Byrne, P. B., \& Burgess, A. 1993, MNRAS, 264, 402

Mihajlov, A. Jevremović, D., Hauschildt, P., Dimitrijević, M. S., Ignjatović, Lj. M., \& Alard, F. 2003, A\&A, 403, 787

Monsignori-Fossi, B., \& Landini, M. 1994, Sol. Phys., 152, 81

Monsignori-Fossi, B., Landini, M., Fruscione, A., \& Dupuis, J. 1995, ApJ, 449,376

Reid, N. I., Hawley, S. L., \& Gizis, J. E. 1995, AJ, 110, 1838

Schmitt, J. H. M. M. 1997, A\&A, 318, 215

Sirk, M. M., Vallerga, J. V., Finley, D. S., Jelinsky, P. J., \& Malina, R. F. 1997, ApJS, 110, 347

Vernazza, J. E., Avrett, E. H., \& Loeser, R. 1981, ApJS, 45, 632

Vriens, L., \& Smeets, A. H. M. 1980, Phys. Rev. A, 22, 940

Worden, S. P., Schneeberger, T. J., Giampapa, M. S., Deluca, E. E., \& Cram, L. E. 1984, ApJ, 276, 270 